\title{
Stabilization of an Unstable L-Leucine Producing Strain of Corynebacterium glutamicum by Enhancement of L-Valine Biosynthesis ${ }^{\dagger}$
}

\author{
Tomoki Azuma and Toshihide NaK ANISHI \\ Technical Research Laboratories, Hofu Plant, Kyowa Hakko Kogyo Co., Lid., \\ Kyowa-machi, Hofu, Yamaguchi 747, Japan
}

Received December 28, 1987

\begin{abstract}
The L-leucine productivity of an L-leucine producer, Corynebacterium glutamicum H-1204, was unstable due to reversion, which was assumed to be caused by the depletion of intracellular L-valine. The genetic enhancement of L-valine biosynthesis was attempted for stabilization using L-valine accumulation as an index. Strain AB-47, derived as an $\alpha$-aminobutyrate resistant mutant, accumulated L-valine as a by-product besides L-leucine. The L-leucine productivity of strain AB-47 was remarkably stabilized under conditions under which that of parental strain $\mathrm{H}-1204$ became unstable. These results indicate that the balanced biosynthesis of metabolites is important for the breeding of a stable amino acid producer.
\end{abstract}

In our previous paper, ${ }^{\text {" }}$ we reported that the L-leucine production by an L-leucine producer, Corynebacterium glutamicum H-1204, was unstable due to reversion. Since the original Lleucine producer (L-type) was found to be a valine leaky mutant, it was strongly suggested that $\alpha$-ketoisovaleric acid, a common precursor of L-leucine and L-valine, was preferentially utilized for L-leucine biosynthesis. This distribution imbalance induced depletion of the intracellular L-valine pool and allowed revertants with higher growth rates to increase in the number.

We also showed that the addition of $L$-valine to the medium was effective for stabilization. ${ }^{2)}$ L-Valine not only promoted the growth of the L-type strain but also inhibited the growth of the revertant (leucine auxotroph), as an antagonist as to its leucine uptake.

In this study, we examined whether or not the genetic enhancement of L-valine biosynthesis through mutagenesis improves the stability of the L-leucine fermentation.

\section{MATERIALS AND METHODS}

Microorganisms. An S-(2-aminoethyl)-L-cysteine resistant mutant, H-1204, derived from Corynebacterium glutamicum, ATCC 13032, was used as the L-leucine producing strain throughout this work. Strain AB-47 was derived from $\mathrm{H}-1204$ as an $\alpha$-aminobutyrate resistant mutant by $N$-methyl- $N^{\prime}$-nitro- $N$-nitrosoguanidine treatment.

Media and culture conditions. The compositions of the media and the culture conditions for test tube and jar fermentor cultivations were given previously. "

Isolation of valine analog resistant mutants. Bacterial cells cultured overnight at $30^{\circ} \mathrm{C}$ in test tubes containing $8 \mathrm{ml}$ of the seed medium were inoculated into $8 \mathrm{ml}$ of the same medium and then cultured for $2 \mathrm{hr}$ at $30^{\circ} \mathrm{C}$. The cells were harvested, washed twice with $50 \mathrm{~mm}$ Tris-maleate buffer ( $\mathrm{pH} \mathrm{6.0)}$ and then resuspended at a concentration of about $10^{9}$ cells $/ \mathrm{ml}$ in an equal volume of the same buffer containing $250 \mu \mathrm{g} / \mathrm{ml}$ of $N$-methyl- $N^{\prime}$-nitro- $N$-nitrosoguanidine (NTG). After incubation for $30 \mathrm{~min}$ at $30^{\circ} \mathrm{C}$, the cells were washed twice and then resuspended in the same buffer. After appropriate dilution, the cell suspension was spread on minimal medium plates containing an analog at a concentration inhibitory for the parental strain. The plates were incubated for 5 to 10 days at $30^{\circ} \mathrm{C}$. The colonies appearing on the plates were isolated as analog resistant mutants and then tested for L-leucine production.

\footnotetext{
$\dagger$ Instability and Stabilization of an L-Leucine Producing Strain of Corynebacterium glutamicum. Part III.
} 
Analysis. The measurement of bacterial growth $\left(\mathrm{OD}_{660}\right)$ and the amount of L-leucine produced was carried out as described previously. "The amount of L-valine was determined by the same method as that for L-leucine.

Chemicals, $\alpha$-Aminobutyrate, $\beta$-hydroxyvaline, DLnorleucine and DL-valine hydroxamate were purchased from Sigma Chemical Co. Amino acids were the products of Kyowa Hakko Kogyo Co., Ltd.

\section{RESULTS AND DISCUSSION}

\section{Enhancement of L-valine biosynthetic activity}

The instability of L-leucine production by strain $\mathrm{H}-1204$ was speculated to be caused by depletion of the intracellular L-valine pool. It was considered essential for stabilization to increase the pool through enhancement of the L-valine biosynthetic activity (Fig. 1).

The biosynthesis is controlled primarily by the first step enzyme, $\alpha$-acetohydroxy acid synthetase, which is sensitive to branched chain amino acids. ${ }^{3)}$ Escherichia coli ${ }^{4}$ and Serratia marcescens ${ }^{5)}$ have been reported to

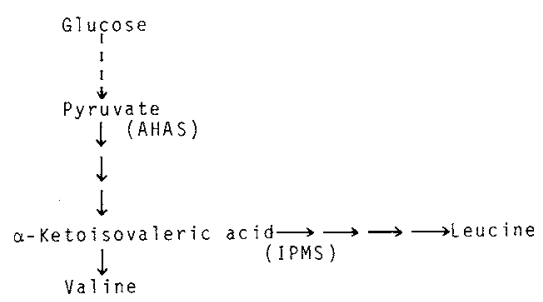

FIG. 1. Metabolic Pathway of L-Leucine and L-Valine Biosynthesis.

Abbreviations: AHAS, $\alpha$-acetohydroxy acid synthetase; IPMS, $\alpha$-isopropylmalate synthetase.

Table I. Summary of the Screening for l-Valine Producers among Mutants Resistant to Various Valine ANALOGS

\begin{tabular}{lrcc}
\hline \multicolumn{1}{c}{ Analog } & $\begin{array}{c}\text { Conc. } \\
(\mathrm{mg} / \mathrm{ml})\end{array}$ & $\begin{array}{c}\text { Number } \\
\text { tested }\end{array}$ & $\begin{array}{c}\text { Number of strains } \\
\text { producing L-valine } \\
\text { as a by-product }\end{array}$ \\
\hline$\alpha$-Aminobutyrate & 20 & 97 & 13 \\
$\beta$-Hydroxyvaline & 10 & 85 & 1 \\
Norleucine & 10 & 45 & 2 \\
Val hydroxamate & 5 & 50 & 1 \\
\hline
\end{tabular}

Cultivations for the screening were performed for $72 \mathrm{hr}$ at $30^{\circ} \mathrm{C}$ in test tubes. excrete L-valine into the media because of the acquirement of valine analog resistance. It has also been reported that $\alpha$-acetohydroxy acid synthetase was derepressed or desensitized as to branched chain amino acids in L-valine producing strains of $S$. marcescens ${ }^{51}$ and Brevibacterium lactofermentum. ${ }^{6}$

Mutants resistant to various valine analogs were derived from strain H-1204 by NTG treatment. These mutants were tested for $\mathrm{L}-$ leucine production. Then, it was determined, from the L-valine accumulation as a byproduct, whether or not the ability of a mutant to synthesize $L$-valine had been enhanced.

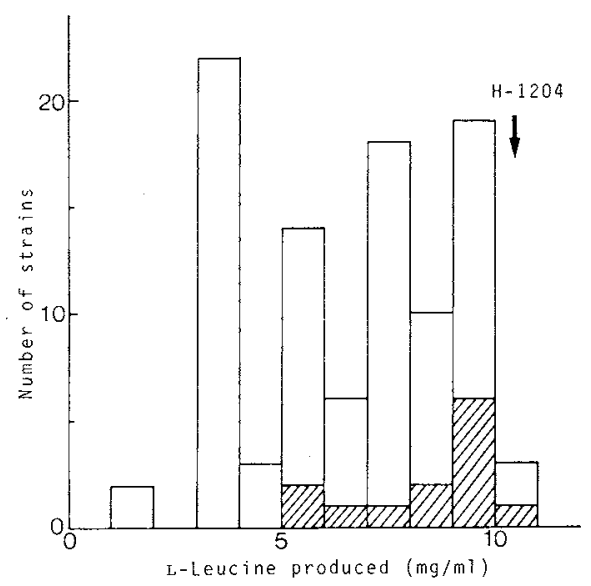

FIG. 2. Distribution of the $\alpha$-Aminobutyrate Resistant Mutants with Respect to L-Leucine and L-Valine Production.

The hatched parts show the numbers of strains producing L-valine as a by-product.

Table H. Relationships between the Production Patterns and the Phenotypes of Representative Strains

\begin{tabular}{ccccc}
\multirow{2}{*}{ Strain } & \multicolumn{2}{c}{ Accumulation $(\mathrm{mg} / \mathrm{ml})$} & & Phenotype \\
\cline { 2 - 3 } & L-Leucine & L-Valine & & Valine \\
\hline H-1204 & 10.1 & 0 & L \\
AB-39 & 10.8 & 0 & \\
AB-47 & 10.2 & 1.1 & L \\
\hline
\end{tabular}

Symbols: L, leaky; +, prototrophic. Strains AB-39 and AB-47 were derived as $\alpha$-aminobutyrate resistant mutants. The phenotypes were determined by replicating each strain on minimal medium with and without L-valine supplementation. 
The results are summarized in Table $I$. Strains exhibiting the simultaneous accumulation of L-leucine and L-valine were found at a higher frequency among the mutants resistant to $\alpha$-aminobutyrate. Figure 2 shows the distribution of the $\alpha$-aminobutyrate resistant mutants with respect to the accumulation of these two amino acids. Among the total 97 mutants tested, two strains showed similar Lleucine productivity to that of the parental strain, H-1204, and 13 strains produced both L-leucine and L-valine.

Relationships between the production patterns and the phenotypes of $\alpha$-aminobutyrate resistant mutants

Strains AB-39 and AB-47 were selected as representatives as to the production patterns and phenotypes (Table II). Strain AB-39, which accumulated an increased amount of Lleucine, still had the same phenotype as the parental strain $\mathrm{H}-1204$ (valine leaky). On the other hand, the phenotype changed from valine leaky to prototrophic in the case of strain AB-47, which accumulated both Lleucine and L-valine. This indicates that the phenotype reflects the ability to synthesize Lvaline.

\section{Stability of $\alpha$-aminobutyrate resistant mutants during subculturing}

The stability of the L-leucine productivity of various strains was examined by subculturing (Table III). The L-leucine production by strains H-1204 and AB-39 decreased remarkably with subculturing. In contrast, strain AB47 showed quite stable productivity. The change to prototrophy was found to be effective for stabilization.

\section{Comparison of L-leucine production by a stable} mutant, $A B-47$, and the parental strain, $H$ 1204

The time courses of L-leucine production were compared for AB-47 and $\mathrm{H}-1204$, in 51 jar fermentors (Fig. 3). Strain H-1204 showed higher growth but only produced $5.2 \mathrm{mg} / \mathrm{ml}$ of L-leucine. Strain AB-47 produced $10.8 \mathrm{mg} / \mathrm{ml}$
Table III. Comparison of Stability during SubCUlTURING

\begin{tabular}{cccc}
\hline \multirow{2}{*}{$\begin{array}{c}\text { Number of } \\
\text { subcultures }\end{array}$} & \multicolumn{3}{c}{ L-Leucine produced $(\mathrm{mg} / \mathrm{ml})$} \\
\cline { 2 - 3 } & H-1204 & AB-39 & AB-47 \\
\hline 0 & 10.1 & 10.8 & 10.2 \\
1 & 7.5 & 8.0 & 10.0 \\
3 & 6.4 & 6.2 & 10.5 \\
5 & 3.7 & 3.9 & 10.3 \\
\hline
\end{tabular}

Subculturing was performed on complete medium slants. The L-leucine production was carried out in test tubes.

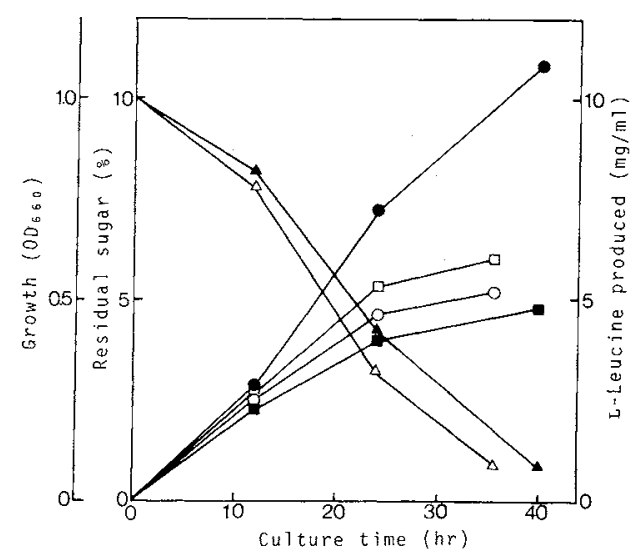

Fici. 3. Comparison of the Time Courses for Strains $H$ 1204 and $\mathrm{AB}-47$.

Symbols: $\bigcirc$, L-leucine produced; $\square \mathbf{\square}$, growth $\left(\mathrm{OD}_{660}\right)$; $\triangle \boldsymbol{\Lambda}$, residual sugar concentration. Open and closed symbols denote $\mathrm{H}-1204$ and $\mathrm{AB}-47$, respectively. The fermentations were carried out in 51 jar fermentors.

of L-leucine stably on $40 \mathrm{hr}$ cultivation under the same conditions. No revertants appeared in the culture broth.

Very few studies have dealt with the stabilization of an amino acid producer on the basis of metabolic analysis. Sugiura and Kisumi ${ }^{\text {) }}$ reported that the depletion of intracellular ATP, which is utilized for histidine biosynthesis, caused the appearance of revertants with reduced L-histidine productivity in $S$. marcescens. They showed that the genetic enhancement of the ATP pool was effective for stabilization of the production and suppression of reversion.

In this study, we also stabilized the L-leucine productivity of strain $\mathrm{H}-1204$. These results 
suggest that one of the reasons for the instability is the metabolic imbalance accompanied by overproduction of metabolites. Therefore, it seems that the maintenance of a metabolic balance is important for the breeding of a stable amino acid producer.

Acknowledgment. The authors are grateful to $\mathrm{Mr}$. Sadao Noguchi for his encouragement throughout this study.

\section{REFERENCES}

1) T. Azuma, T. Nakanishi and H. Hagino, Agric. Biol. Chem., 51, 3245 (1987).

2) T. Azuma, T. Nakanishi and H. Hagino, Agric. Biol. Chem., 52, 1129 (1988).

3) J. M. Blett, W. J. Pledger and H. E. Umbarger, Biochem. Biophys. Res. Commun., 48, 444 (1972).

4) O. Karlstrom, Biotechnol. Bioeng., 7, 245 (1965).

5) M. Kisumi, S. Komatsubara and I. Chibata, Amino Acid and Nucleic Acid, 19, 1 (1969).

6) T. Tsuchida and H. Momose, Agric. Biol. Chem., 39, 2193 (1975).

7) M. Sugiura and M. Kisumi, Appl. Environ. Microbiol., 48, 43 (1984). 